\title{
Stabilization and structure of telomeric and c-myc region intramolecular G-quadruplexes: The role of central cations and small planar ligands
}

\author{
Valérie Gabelica ${ }^{1}$, Erin Shammel Baker ${ }^{2}$, Marie-Paule Teulade-Fichou ${ }^{3}$, Edwin De Pauw ${ }^{1}$, \\ Michael T. Bowers ${ }^{2 *}$
}

\begin{abstract}
A contribution from ${ }^{1}$ the Department of Chemistry, University of Liège, Belgium, ${ }^{2}$ the Department of Chemistry and Biochemistry, University of California at Santa Barbara, USA, and ${ }^{3}$ the Collège de France, Paris, France.
\end{abstract}

\section{Supporting Information Contents:}

Full author list of reference 59

Full Scan ESI-MS spectra discussion

Figure S1: Typical full scan MS spectra obtained for the oligonucleotides. (p.S3)

2 ns modeling of $[\mathrm{Pu} 27]^{6-}$ in the mostly parallel conformation

Figure S2: Modeling with and without ammonium cations.

Determination of ligand binding mode 
Complexes of the Pu27 with TmPyP4

(p.S5)

Figure S3: Modeling of $[\mathrm{Pu} 27+\mathrm{TmPyP} 4]^{5-}$

Figure S4: ATD of [Pu27+TmPyP4] $]^{6-}$

Full set of data with $\mathbf{T}_{\mathbf{3 . 5}}, \mathbf{T}_{\mathbf{6}}$ and $\mathbf{P u 2 2}$

Table S1. Cross sections of $\left[\mathbf{T}_{\mathbf{3 . 5}}+1 \text { Ligand }\right]^{4-}$

Table S2. Cross sections of $\left[\mathbf{T}_{\mathbf{3 . 5}}+1 \text { Ligand }\right]^{5-}$

Table S3. Cross sections of $\left[\mathbf{T}_{\mathbf{6}}+1 \text { Ligand }\right]^{6-}$

Table S4. Cross sections of $[\mathbf{P u} 22+1 \text { Ligand }]^{5-}$

Table S5. Cross sections of $[\mathbf{P u} 22+2 \text { Ligands }]^{5-}$

(p.S13)

Figure S5. Structures of Pu22 + MMQ1, MMQ3 and BOQ1

(p.S14)

References for the supporting information

(p.S15)

\section{Full author list for Reference 59:}

Case, D. A.; Perlman, D. A.; Caldwell, J. W.; Cheatham, T. E., III; Wang, J.; Ross, W. S.;

Simmerling, C. L.; Darden, T. A.; Merz, K. M.; Stanton, R. V.; Cheng, A. L.;

Vincent, J. J.; Crowley, M.; Tsui, V.; Gohlke, H.; Radmer, R. J.; Duan, Y.; Pitera,

J.; Massova, I.; Seibel, G. L.; Singh, U. C.; Weiner, P. K.; Kollman, P. A. AMBER

7; University of California, San Francisco, 2002. 


\section{Full Scan ESI-MS spectra: detailed discussion}
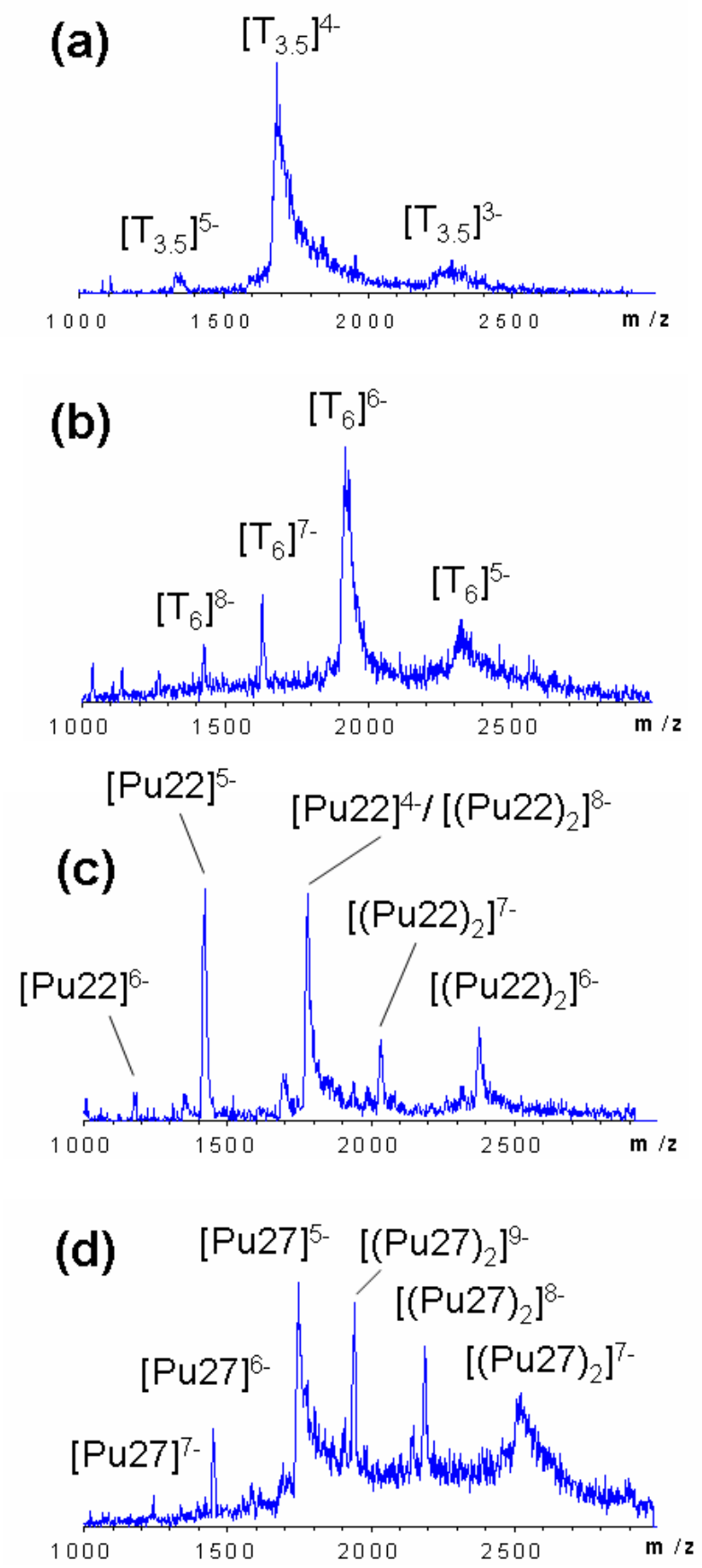

Figure S1. Typical full scan MS spectra obtained for the oligonucleotides. Assignment to monomers/dimers for even monomer charge states was based on ion mobility data. 
In the case of the two sequences derived from the promoter of c-myc, the full scan mass spectra are complicated by the appearance of dimers in addition to the monomer peaks. This did not constitute a problem for cross section determination because mixtures of monomer and dimer at a given mass-to-charge ratio are easily resolved in the ion mobility experiments due to differences in drift times. The dimer (twice the charge, twice the mass, but less than twice the collision cross section) moves faster in the drift cell. For clarity, only arrival time distributions with no dimer interference are shown.

\section{2 ns modeling of $[\mathrm{Pu} 27]^{6-}$ in the mostly parallel conformation}

After the publication of the crystallographic structure of the analog $\mathrm{Pu} 24 \mathrm{I}$, we also performed 2 ns modeling on a mixed (mostly parallel) structure of Pu27 in the absence and in the presence of ammonium ions. The results (Figure S2) show that the structure is not as stable as the fully parallel structure (see Figure 4 in paper). The presence of two ammonium ions delay the unfolding, but does not prevent it on the 2 ns time scale.
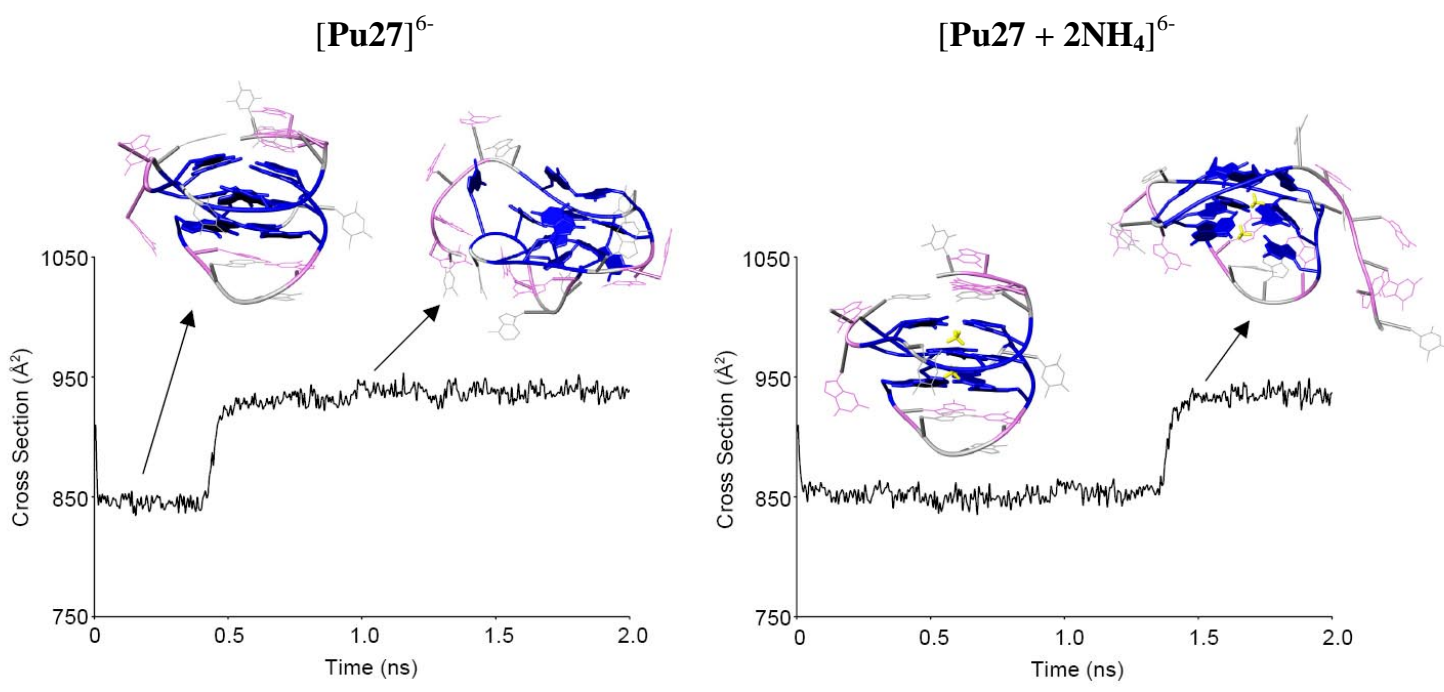

Figure S2. Molecular dynamics modeling of $[\mathbf{P u 2 7}]^{6-}($ left $)$ and $\left[\mathbf{P u 2 7}+2 \mathrm{NH}_{4}\right]^{6-}$ (right). 


\section{Determination of ligand binding mode}

For all charge states for which we found an intact folded G-quadruplex conformation for the oligonucleotide alone, the complexes with ligands always show a single peak in their arrival time distribution. To interpret the experimental collision cross sections in terms of structure, we calculated the theoretical cross sections for three possible binding modes (intercalation, end stacking, and nonspecific external binding) and each of the three major G-quadruplex folds (parallel, antiparallel, and mixed conformations represented in Scheme 1).

\section{Complexes of the Pu27 with TmPyP4}

The case of $[\mathbf{P u} 27+\mathrm{TmPyP} 4]^{5-}$, for which a collision cross section of $841 \AA^{2}$ was measured, will be described in detail. We were particularly interested in this complex because ligandinduced conformational transition from a parallel to an antiparallel G-quadruplex was reported for this ligand and the myc sequence ${ }^{1,2}$. Theoretical cross sections and the corresponding structures are collected Figure S3. The experimental collision cross section matches best with the structure of the complex between the parallel G-quadruplexes and either an end-stacked or an intercalated ligand. The cross sections do not allow differentiating the fully parallel (propeller) conformation from the mixed, but mostly parallel conformation. A transition to an antiparallel conformation would have been characterized by a transition to a larger collision cross section (with a $\sigma(\mathrm{CCS})$ of $50 \AA^{2}$ ). It must be noted that the conformational transition reported previously ${ }^{4}$ was observed in the absence of salts except the added TmPyP4, while our experiments were conducted in the presence of $20 \mathrm{mM}$ or $150 \mathrm{mM}$ ammonium acetate. The cross section alone does not allow making a difference between end stacking and intercalation mode. However the end stacked structures are always at least $20 \mathrm{kcal} / \mathrm{mol}$ lower in energy than the intercalated structures. 
The ATD for $[\mathbf{P u 2 7}]^{6-}$ shows a bimodal distribution (Figure S4), and the comparison with the oligonucleotide alone shows that the contribution of the more extended structure with one quartet broken is lower in the presence of ligand. Like for charge state 5-, there is no indication of ligand-induced conformational change of the G-quadruplex. 
is
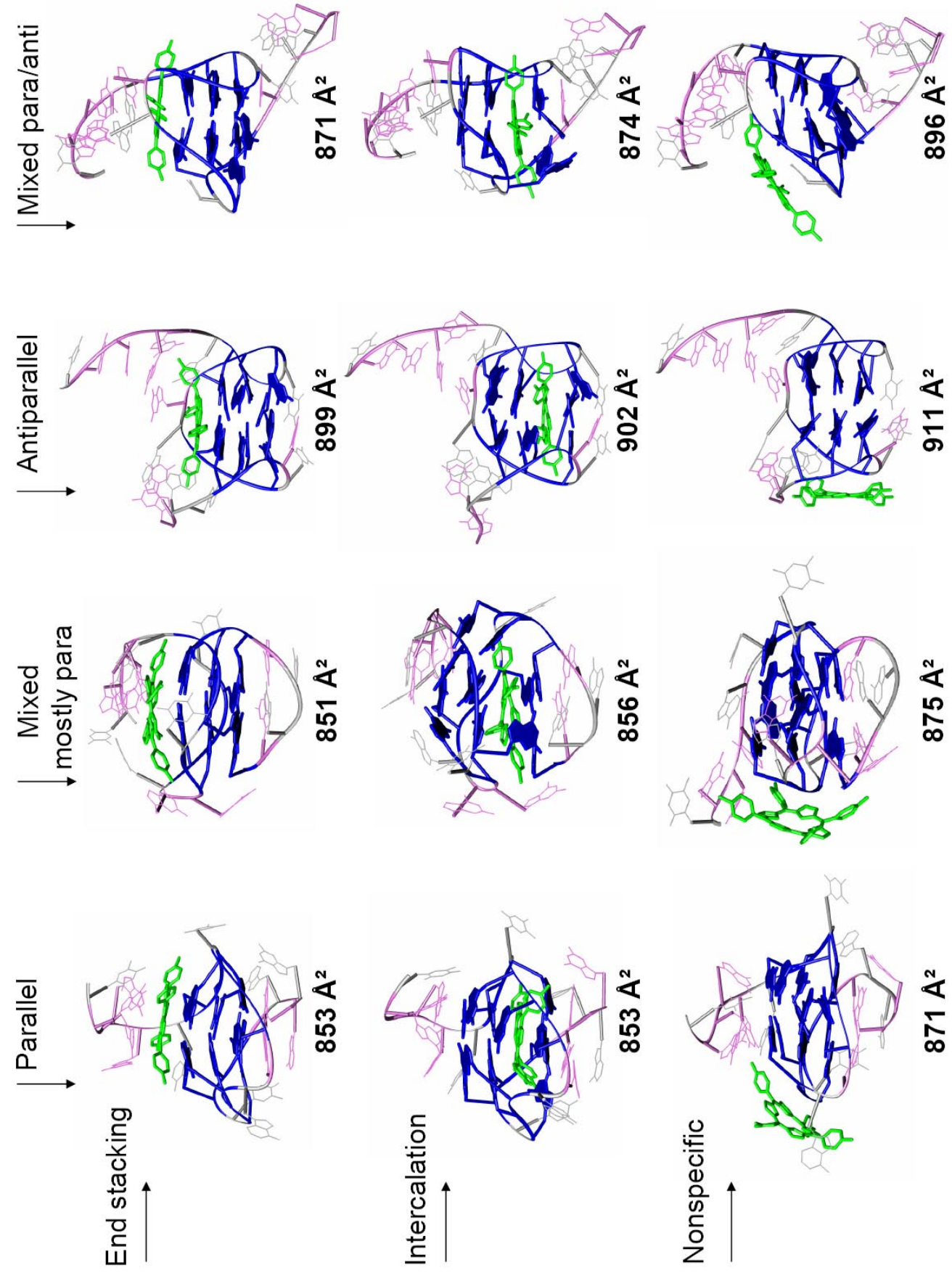

总 春

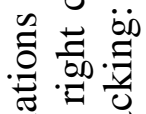

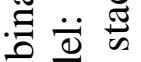

है ซี

올 윻

후월

的䨔

링

产葛

ิิ

$\times$

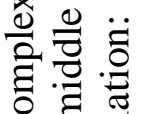

跑

离离

造.

壱

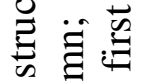

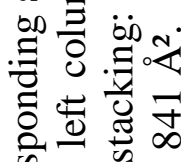

过

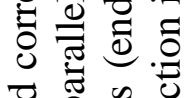

ప

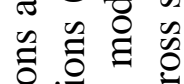

.웡

क छ

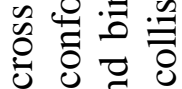

స్

월워

氮

छ항

ن

त \&

늘

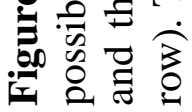



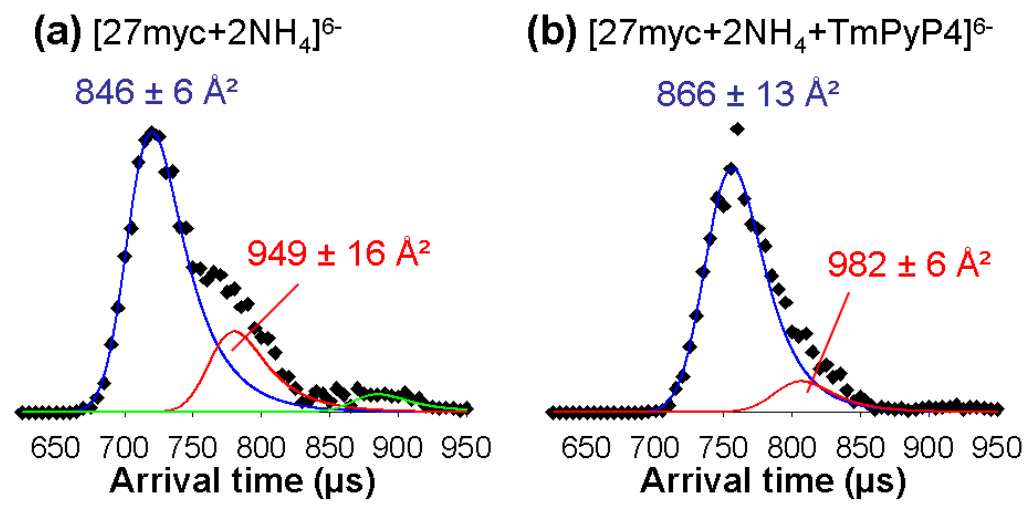

Figure S4. Comparison of the arrival time distribution of (a) [Pu27 + $\left.2 \mathrm{NH}_{4}\right]^{6-}$ and (b) $\left[\mathbf{P u 2 7}+2 \mathrm{NH}_{4}+\mathrm{TmPyP} 4\right]^{6-}$.

Complete set of data for the complexes with the oligonucleotides $\mathbf{T}_{\underline{3.5}} \mathbf{T}_{6} \underline{\text { and }} \mathbf{P u 2 2}$.

All data are gathered in Tables S1 and S2 for $\mathbf{T}_{\mathbf{3 . 5}}$, Table S3 for $\mathbf{T}_{\mathbf{6}}$, and Tables S4 and S5 for Pu22. Selected theoretical structures of the complexes of Pu22 and ligands MMQ1, MMQ3 and BOQ1 are shown in Figure S5. 


\begin{tabular}{|c|c|c|c|c|c|c|c|}
\hline \multirow{10}{*}{ 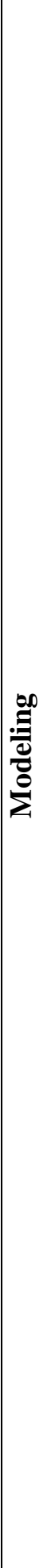 } & $\begin{array}{l}\frac{\vec{J}}{\bar{z}} \\
\frac{0}{0} \\
\end{array}$ & 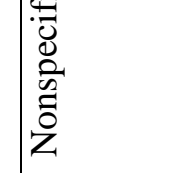 & 売 & शे & ָิ & $\stackrel{8}{2}$ & $\frac{\infty}{2}$ \\
\hline & \multirow{3}{*}{ 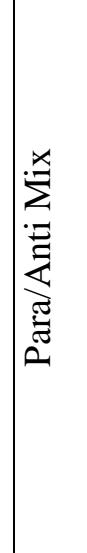 } & 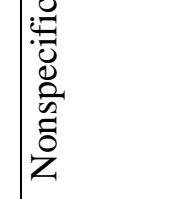 & $\stackrel{\circ}{\infty}$ & $\frac{t}{\infty}$ & ఏి & $\infty$ & ळे \\
\hline & & 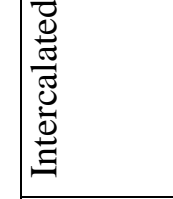 & $\vec{\Omega}$ & 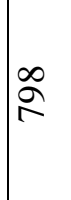 & $\vec{\infty}$ & $\bar{\Omega}$ & 只 \\
\hline & & 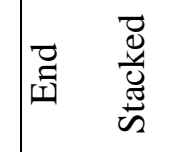 & হి & $\bar{\Omega}$ & 榙 & $\stackrel{\infty}{\stackrel{\infty}{\infty}}$ & $\stackrel{\bigotimes}{\circledR}$ \\
\hline & \multirow{3}{*}{ 惫 } & 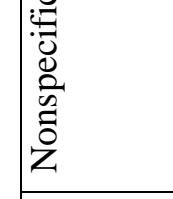 & 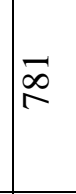 & 文 & $\frac{n}{\infty}$ & $\stackrel{\text { }}{\stackrel{I}{\Sigma}}$ & $\mathbb{\infty}_{\infty}^{\infty}$ \\
\hline & & 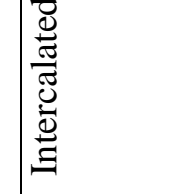 & है & $\stackrel{\vec{\imath}}{\imath}$ & $\vec{\infty}$ & న̂ & in \\
\hline & & 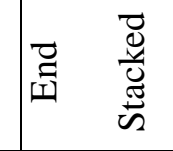 & స్ర & $\vec{\imath}$ & $\stackrel{\infty}{\infty}$ & 电 & $\begin{array}{l}\infty \\
\substack{\infty \\
n}\end{array}$ \\
\hline & \multirow{3}{*}{ 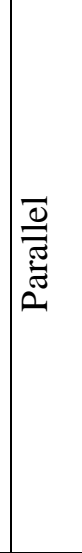 } & 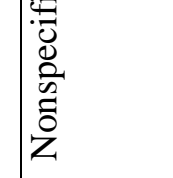 & $\widetilde{\infty}$ & 倠 & 命 & గ్రి & $\infty_{\infty}^{\infty}$ \\
\hline & & 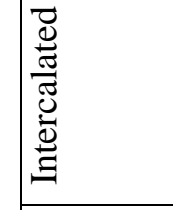 & ळे & $\bar{\infty}$ & $\vec{\infty}$ & 冓 & $\infty_{\infty}^{\infty}$ \\
\hline & & 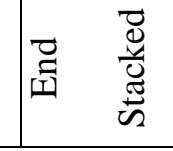 & $\mathscr{\infty}_{\infty}$ & $\widehat{\infty}$ & దे & న్ & 凩 \\
\hline \multirow[t]{2}{*}{ 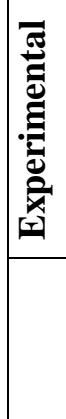 } & & & & I' & $\begin{array}{l}\qquad \\
\text { 导 }\end{array}$ & $\begin{array}{l}\Xi \\
\text { I }\end{array}$ & $\begin{array}{l}\widehat{c} \\
y \\
y \\
+1 \\
\infty \\
c \\
c\end{array}$ \\
\hline & & & 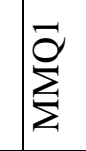 & $\sum_{\Sigma}^{2}$ & $\bar{D}$ & 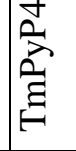 & 畄窟 \\
\hline
\end{tabular}


के

\begin{tabular}{|c|c|c|c|c|c|c|c|c|}
\hline \multirow{10}{*}{$\frac{0.00}{: 0}$} & $\begin{array}{l}\frac{z}{3} \\
\bar{\Xi} \\
\frac{0}{0} \\
0\end{array}$ & 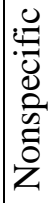 & & ஜ & $\bar{\nabla}$ & 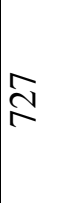 & $\stackrel{2}{2}$ & $\stackrel{2}{2}$ \\
\hline & \multirow{3}{*}{ 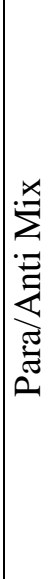 } & \multicolumn{2}{|c|}{ 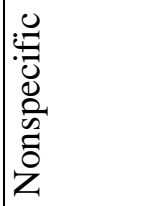 } & $\stackrel{\varnothing}{ }$ & $\frac{0}{\infty}$ & $\underset{\infty}{\infty}$ & $\bar{\infty}$ & $\frac{\nabla}{\infty}$ \\
\hline & & \multicolumn{2}{|l|}{ 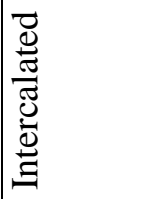 } & পৃ & ஓ & $\begin{array}{l}\infty \\
N \\
\infty\end{array}$ & $\stackrel{\Re}{\gamma}$ & $\bar{a}$ \\
\hline & & & 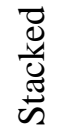 & $\stackrel{\Omega}{\gamma}$ & $\stackrel{+}{2}$ & $\grave{\infty}$ & হ & 2 \\
\hline & & 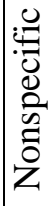 & & $\infty$ & $\overline{2}$ & $\frac{\Gamma}{\infty}$ & $\underset{\infty}{\infty}$ & $\infty$ \\
\hline & 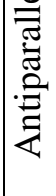 & 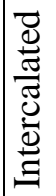 & & $\ddot{2}$ & 2 & $\frac{n}{\infty}$ & $n$ & $\stackrel{8}{2}$ \\
\hline & & 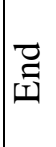 & 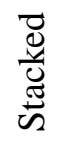 & $\frac{1}{2}$ & $\stackrel{0}{2}$ & ஓి & In & in \\
\hline & & 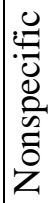 & & $\ddot{n}$ & $\sqrt[n]{n}$ & $\begin{array}{l}\infty \\
\infty \\
\infty\end{array}$ & $\hat{n}$ & $\bar{\infty}$ \\
\hline & 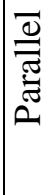 & 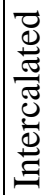 & & $\underset{\Phi}{\Im}$ & 㐫 & $\frac{\infty}{\infty}$ & $\underset{\infty}{\nexists}$ & $\underset{\infty}{\infty}$ \\
\hline & & & $\begin{array}{l}\vec{d} \\
\frac{\ddot{d}}{\tilde{W}} \\
\text { in }\end{array}$ & 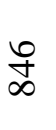 & $\underset{\infty}{\stackrel{\Phi}{ }}$ & $\underset{\infty}{\infty}$ & $\hat{\infty}$ & $\underset{\infty}{\ddagger}$ \\
\hline & & & & $\begin{array}{l}\widehat{d} \\
0 \\
+1 \\
0 \\
0 \\
2\end{array}$ & $\begin{array}{l}\overparen{d} \\
\sim \\
+1 \\
N \\
\end{array}$ & $\begin{array}{l}\stackrel{\Xi}{2} \\
2\end{array}$ & 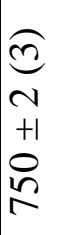 & $\begin{array}{l}\widehat{d} \\
m \\
+1 \\
n \\
n \\
r\end{array}$ \\
\hline & & & & $\underset{\sum}{\overline{0}}$ & $\stackrel{\infty}{\sum}$ & $\bar{\varnothing}$ & 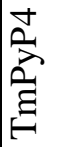 & $\frac{\frac{\alpha}{10}}{\frac{1}{2}}$ \\
\hline
\end{tabular}


के

\begin{tabular}{|c|c|c|c|c|c|c|}
\hline 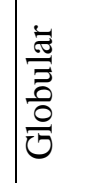 & 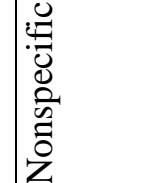 & $\cong$ & 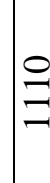 & $\stackrel{\Xi}{\Xi}$ & $\stackrel{\varrho}{ٍ}$ & 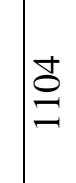 \\
\hline & 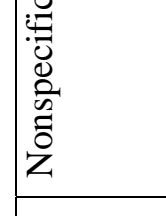 & 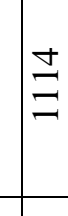 & 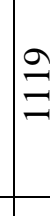 & 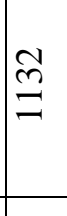 & $\stackrel{\varrho}{\Xi}$ & $\equiv$ \\
\hline 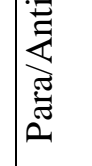 & 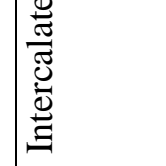 & 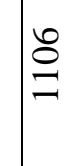 & 号 & $\stackrel{\circledast}{\cong}$ & ఏٍ & $\stackrel{\partial}{\Xi}$ \\
\hline & 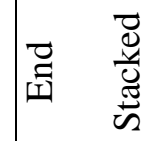 & 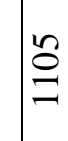 & $\supseteqq$ & I & है & 声 \\
\hline & 悹 & $\frac{2}{8}$ & 总 & 高 & 莺 & gి \\
\hline 咅 & 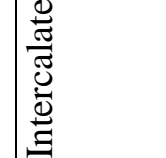 & $\stackrel{\mathscr{0}}{\stackrel{g}{g}}$ & $\stackrel{g}{g}$ & ถ્ּ & 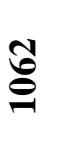 & $\overrightarrow{\underline{g}}$ \\
\hline & 畐咅 & 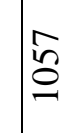 & ğ & $\underline{\underline{E}}$ & Б大 & 兽 \\
\hline & 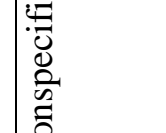 & $\stackrel{d}{\cong}$ & $\Xi$ & 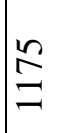 & 常 & 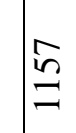 \\
\hline 产 & 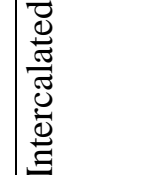 & $\stackrel{\bar{g}}{\underline{2}}$ & 㘿 & 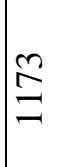 & 影 & $\exists$ \\
\hline & 胥 & 导 & I & 를 & İ & $\stackrel{2}{g}$ \\
\hline & & & & & & $\bar{z}_{\bar{z}}$ \\
\hline & & & & $\overline{\bar{g}}$ & 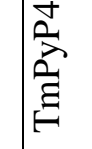 & \\
\hline
\end{tabular}


के

\begin{tabular}{|c|c|c|c|c|c|c|c|c|}
\hline \multirow{10}{*}{ 照 } & $\begin{array}{l}\frac{\vec{t}}{\Xi} \\
\frac{0}{0} \\
0\end{array}$ & $\begin{array}{l}0 \\
0 \\
0 \\
0 \\
0 \\
0 \\
0 \\
0 \\
z \\
Z\end{array}$ & & $\bar{a}$ & $\underset{\infty}{\infty}$ & $\infty$ & $\infty$ & $\frac{\infty}{\infty}$ \\
\hline & \multirow{3}{*}{ 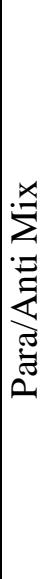 } & \multicolumn{2}{|l|}{$\begin{array}{l}0 \\
0 \\
0 \\
0 \\
0 \\
0 \\
\tilde{z} \\
0 \\
z\end{array}$} & $\underset{\infty}{\mathbb{D}}$ & $\stackrel{5}{\infty}$ & $\frac{ \pm}{\infty}$ & $\mathcal{O}_{\infty}$ & $\stackrel{\infty}{\infty}$ \\
\hline & & \multicolumn{2}{|l|}{ 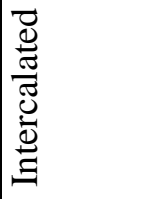 } & $\begin{array}{l}\infty \\
\infty \\
\infty\end{array}$ & $\infty$ & $\stackrel{8}{\infty}$ & $\stackrel{\infty}{\infty}$ & $\stackrel{\infty}{\infty}$ \\
\hline & & & $\begin{array}{l}\frac{\vec{Q}}{\mathscr{U}} \\
\frac{\pi}{\tilde{N}}\end{array}$ & $\infty$ & $\underset{\infty}{\infty}$ & $\frac{2}{2}$ & $\frac{2}{2}$ & $\underset{\infty}{\infty}$ \\
\hline & & 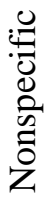 & & $\frac{\infty}{\infty}$ & $\frac{0}{\infty}$ & $\vec{\infty}$ & $\frac{m}{\infty}$ & ஓे \\
\hline & 疍 & 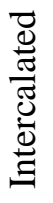 & & $\stackrel{\infty}{\infty}$ & $\stackrel{8}{\infty}$ & in & $\stackrel{2}{2}$ & $\stackrel{2}{2}$ \\
\hline & & 空 & 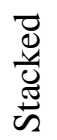 & ஓ & ং & $\bar{\varpi}$ & 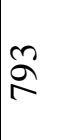 & $\bar{\Omega}$ \\
\hline & & 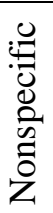 & & ๙ু & $\frac{n}{2}$ & $\stackrel{8}{\infty}$ & $\stackrel{\infty}{\infty}$ & $\stackrel{\infty}{\infty}$ \\
\hline & 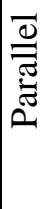 & 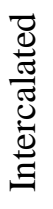 & & $\ddot{\infty}$ & 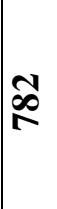 & ஓ & $\underset{⿱}{*}$ & $\stackrel{2}{2}$ \\
\hline & & 胥 & 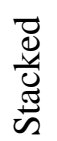 & $\stackrel{R}{R}$ & $\mathbb{N}$ & $\infty$ & $\frac{8}{2}$ & 12 \\
\hline 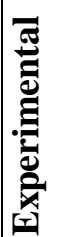 & & & & \begin{tabular}{l}
$\widehat{0}$ \\
\hdashline \\
+1 \\
$\infty$ \\
$\infty$ \\
$\infty$
\end{tabular} & $\begin{array}{l}\widehat{0} \\
n \\
+1 \\
\hat{\sigma} \\
2\end{array}$ & \begin{tabular}{l}
$\overparen{d}$ \\
\hdashline+1 \\
+1 \\
$\stackrel{2}{2}$
\end{tabular} & 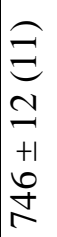 & 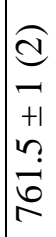 \\
\hline & & & & $\sum_{\Sigma}^{\bar{O}}$ & $\sum_{\sum}^{\infty}$ & $\bar{\sigma}$ & 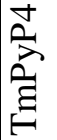 & $\frac{\frac{1}{w}}{\frac{1}{2}}$ \\
\hline
\end{tabular}


$\frac{m}{n}$

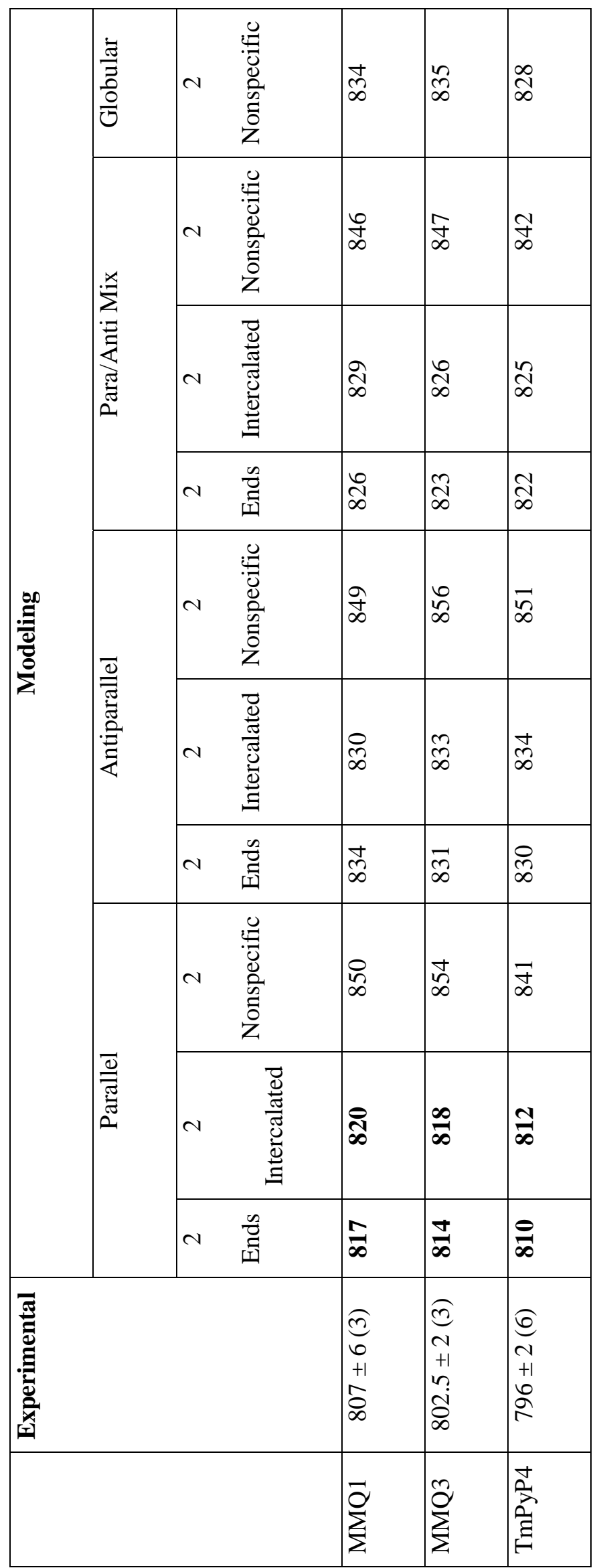


$\frac{t}{n}$

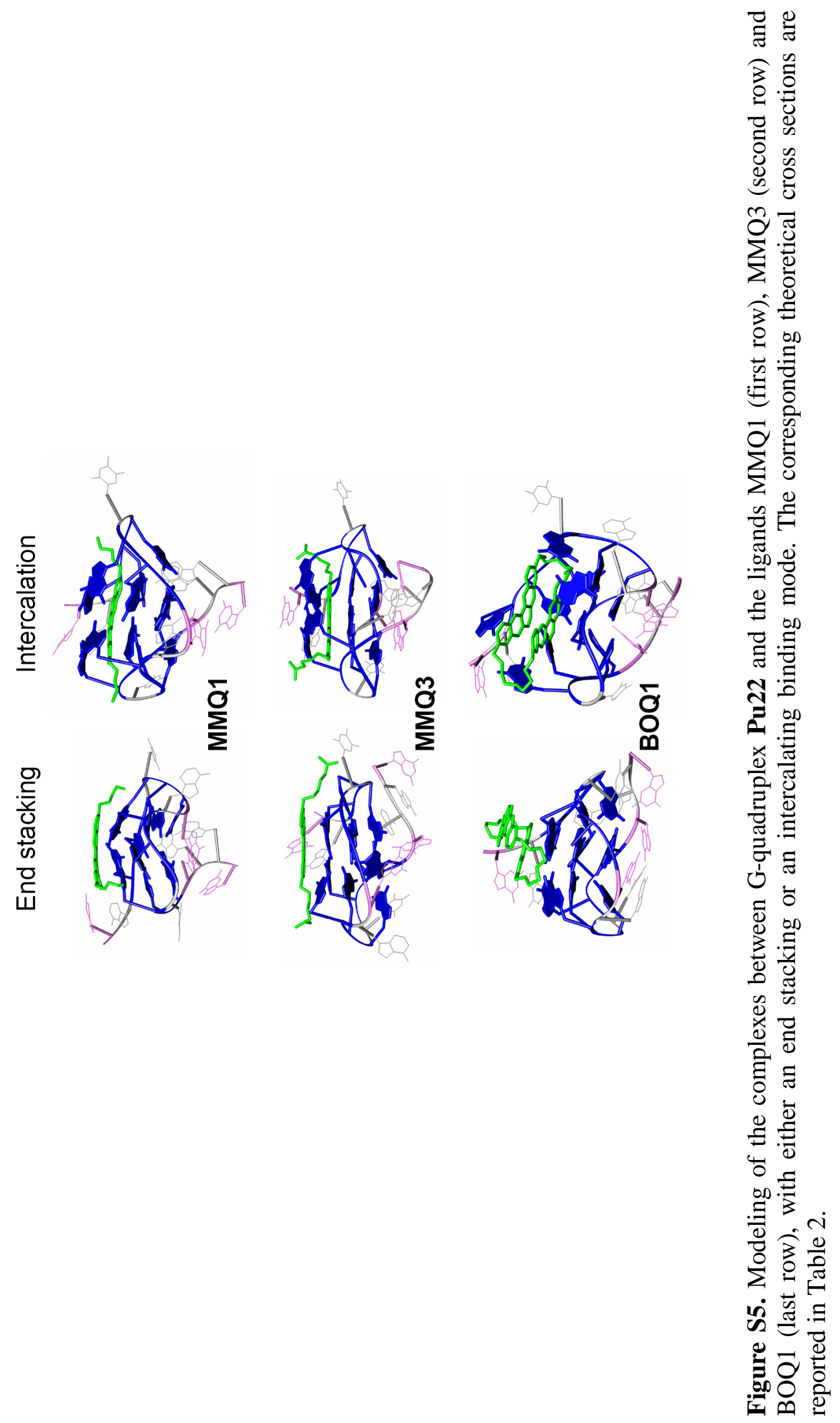




\section{References}

1. Seenisamy, J.; Rezler, E. M.; Powell, T. J.; Tye, D.; Gokhale, V.; Joshi, C. S.;

Siddiqui-Jain, A.; Hurley, L. H. The dynamic character of the G-quadruplex element in the c-MYC promoter and modification by TMPyP4. J. Am. Chem. Soc. 2004, 126, 8702-8709.

2. Rezler, E. M.; Seenisamy, J.; Bashyam, S.; Kim, M. Y.; White, E.; Wilson, W. D.; Hurley, L. H. Telomestatin and diseleno sapphyrin bind selectively to two different forms of the human telomeric G-quadruplex structure. $J$. Am. Chem. Soc. 2005, 127 (26), 9439-9447. 